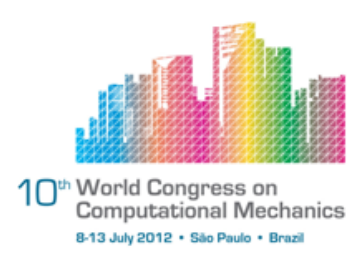

\title{
NONLOCAL DAMAGE MODEL USING THE MESHLESS FINITE POINTS METHOD
}

\author{
F. Chacana Yorda ${ }^{1}$, L. Pérez Pozo ${ }^{2}$ \\ ${ }^{1}$ Department of Mechanical engineering, Universidad Técnica Federico Santa María, Val- \\ paraíso Chile (felipe.chacana@alumnos.usm.cl) \\ ${ }^{2}$ Department of Mechanical engineering, Universidad Técnica Federico Santa María, Val- \\ paraíso Chile (luis.perez@usm.cl)
}

Abstract. We present the formulation and application for a nonlocal model with gradi-

ent type regularization incorporated into the model by means of non-local displacements.

The strong formulation of the FPM allows the use of high-order differentially shape functions with which we can approximate directly the fields of the nonlocal displacements, for that this technique is very attractive for a computational viewpoint.

For the numerical implementation we used a fully explicit integration scheme and for the nonlinear problem the Newton Raphson iterative scheme. The validation of the obtained results is made starting from typical benchmark problems and available results on associated literature.

Keywords: Non-locas damage models. Meshless. gradient models.

\section{INTRODUCTION}

The finite points method (FPM) was proposed by Oñate et al $[5,6]$ initially with the purpose of solving convective transport and fluid flow problems. Later, its application was extended to advection-diffusion transport [7] and incompressible flow problems [8]. In the context of solid mechanics, FPM has been applied successfully in elasticity [9, 20, 10, 21], solid dynamics [11] and non-linear material behavior problems [13]. The non-dependence on a mesh or integration procedures is an important aspect which transform the FPM in a truly meshless method.

The continuous damage models may be employed to describe the evolution of failure processes between the undamaged state and macroscopic crack initiation [23]. Regarding numerical simulations, this presents pathological mesh sensitivity, in that way different solutions have been proposed in the literature to remedy this physically unrealistic behavior by means cohesive crack models [24], Crack bands model [25] and regularized models[26]. In this work we focus on the regularized models via non-local effects, incorporated a material 
characteristic length and the implicit gradient-enhanced continuum model based on non-local displacements [27].

For the gradient-enhanced model the strong formulation of the FPM allows the use of high-order differentially shape functions with which we can approximate directly the fields of local and non-local displacements [28].

This paper contains: Section 2, The basic formulation of the FPM is presented. Section 3 The nonlocal damage model via nonlocal displacements. Section 4 describe the numerical implementation. Section 5 show the clasic numerical examples, this examples are in 1D and 2D.

\section{The finite points method}

Although the FPM introduced by Oñate et al. was originally formulated for the numerical solution of convective transport and fluid flow problems, it can be easily adapted to the Stress equation. In this section, we review the basic formulation of the FPM and provide a brief overview of its main features. In order to obtain the final system of discrete equations, the FPM approximates the local solution of a partial differential equation in each point of the discretized domain by means of a weighted least squares technique and a point collocation procedure. Due to the local character of the approximation procedure used by this method, it is necessary to define a sub-domain for each node, $\Omega_{k}$, that contain neighboring nodes selected by a suitable criterion $[18,9]$. This collection of points is called a cloud, and its referential central point is the star node. For example, a relevant aspect in the definition of clouds is that their superposition must produce the whole domain, $\Omega$.

$$
\bigcup_{k=1}^{N_{p}} \Omega_{k}=\Omega,
$$

where $N_{p}$ is the total number of nodes. Note that the definition of clouds is the basic, initial step in implementing the FPM approximation using fixed weighted least squares. With the discretized domain defined, let us define a function $u(x)$, which is locally approximated by $\hat{u}(x)$ only valid in the cloud $\Omega_{k}$ associated with the star node $x_{k}$ as a linear combination of known functions $\boldsymbol{p}(x)$.

$$
u(x) \cong \hat{u}(x)=\boldsymbol{p}(x) \boldsymbol{\alpha}_{\boldsymbol{k}} \quad \forall x \in \Omega_{k},
$$

where $\boldsymbol{p}(x)$ is the vector that represents the basis of $m$ linearly independent functions, and $\boldsymbol{\alpha}_{\boldsymbol{k}}$ is a vector of constant parameters only valid in $\Omega_{k}$. The elements of the interpolation base may belong to any function family. Nevertheless, for simplicity, the $m$ first monomial polynomials are used. Since Eq. (2) is valid for all $N_{c}$ points of the $k$-th sub-domain, the approximations $\hat{u}(\boldsymbol{X})$ conform to a Vandermonde system given by the following relation.

$$
u\left(X^{k}\right) \cong \hat{u}\left(X^{k}\right)=P\left(X^{k}\right) \cdot \alpha_{k},
$$

where

$$
\begin{aligned}
& \boldsymbol{X}^{\boldsymbol{k}}=\left[x_{k, 1} \ldots x_{k, N_{c}}\right]^{T}, \quad \boldsymbol{u}\left(\boldsymbol{X}^{\boldsymbol{k}}\right)=\left[u\left(x_{k, 1}\right) \ldots u\left(x_{k, N_{c}}\right)\right]^{T}, \\
& \hat{\boldsymbol{u}}\left(\boldsymbol{X}^{\boldsymbol{k}}\right)=\left[\hat{u}\left(x_{k, 1}\right) \ldots \hat{u}\left(x_{k, N_{c}}\right)\right]^{T}, \quad \boldsymbol{\alpha}_{\boldsymbol{k}}=\left[\alpha_{k, 1} \ldots \alpha_{k, N_{c}}\right]^{T},
\end{aligned}
$$


and

$$
\boldsymbol{P}\left(\boldsymbol{X}^{\boldsymbol{k}}\right)=\left[\begin{array}{c}
\boldsymbol{p}\left(x_{k, 1}\right) \\
\vdots \\
\boldsymbol{p}\left(x_{k, N_{c}}\right)
\end{array}\right] .
$$

In general, the number of points $N_{c}$ that conform to the cloud is greater than the number of functions $m$ that define the basis; hence, the matrix $P\left(X^{\boldsymbol{k}}\right)$ is usually rectangular. This means that the property of interpolation is lost, and the problem must be addressed with numerical approximation. The coefficients of the vector $\alpha_{k}$ must be determined in such a way that the weighted sums of the squared differences between the exact values $u(x)$ and the approximated values $\hat{u}(x)$ of each point is minimized according to the following equation.

$$
\min \left\{\sum_{j=1}^{N_{c}} w\left(x_{j}\right) \cdot\left(u\left(x_{j}\right)-\hat{u}\left(x_{j}\right)\right)^{2}\right\},
$$

where $w\left(x_{j}\right)$ is a fixed weighting function defined in $\Omega_{k}$ and evaluated for the node $x_{j}$. See refs. $[5,6]$. The minimization process described by Eq. (4) leads to the following expression for vector $\alpha_{k}$.

$$
\alpha_{k}=A^{-1}\left(X^{k}\right) B\left(X^{k}\right) \lambda\left(X^{k}\right),
$$

where $\lambda\left(X^{\boldsymbol{k}}\right)$ is a vector that represents the unknown parameters sought on the cloud $\Omega_{k}$ defined as follows.

$$
\boldsymbol{\lambda}\left(\boldsymbol{X}^{\boldsymbol{k}}\right)=\left[\lambda\left(x_{k, 1}\right) \ldots \lambda\left(x_{k, N_{c}}\right)\right]^{T},
$$

Additionally, matrix $A\left(X^{k}\right), B\left(X^{k}\right)$ and $W\left(X^{k}\right)$ are given as follows.

$$
A\left(X^{\boldsymbol{k}}\right)=\boldsymbol{P}\left(X^{\boldsymbol{k}}\right) W\left(X^{\boldsymbol{k}}\right) \boldsymbol{P}^{T}\left(X^{\boldsymbol{k}}\right), \quad \boldsymbol{B}\left(X^{\boldsymbol{k}}\right)=\boldsymbol{P}^{T}\left(X^{\boldsymbol{k}}\right) \boldsymbol{W}\left(X^{\boldsymbol{k}}\right),
$$

and $W\left(X^{k}\right)$ is an $N_{c} \times N_{c}$ diagonal matrix defined by:

$$
W\left(X^{\boldsymbol{k}}\right)=\left[\operatorname{diag}\left(w\left(x_{k, 1}\right) \ldots w\left(x_{k, N_{c}}\right)\right)\right],
$$

where the weighting functions $w\left(x_{j}\right)$ are derived in order to have unit values near the star node and zero values outside the $\Omega_{k}$ sub-domains. Under the FPM, the common selection is the normalized Gaussian given as follows.

$$
w\left(x_{j}\right)= \begin{cases}\frac{\exp \left(-\left(h_{j} / \zeta\right)\right)-\exp (-(r / \zeta))}{(1-\exp (-(r / \zeta))} & \text { if } h_{j} \leq r \\ 0 & \text { if } h_{j}>r\end{cases}
$$

where $h_{j}$ is the distance between the star node, the point $x_{j}, r=q \cdot h_{\max }\left(\max\right.$. of $h_{j}$ ) is a reference distance, and $\zeta=\beta \cdot r$. A detailed description of the effects of the constant parameters $q$ and $\beta$ on numerical approximation as well as guidelines for setting their values are presented in [19]. Other considerations in selecting the function $w\left(x_{j}\right)$ can be found in refs. [6, 5, 20, 22]. Finally, replacing Eq. (5) in (2), the next relation is obtained.

$$
\hat{u}(x)=N^{T}(x) \boldsymbol{\lambda}\left(X^{\boldsymbol{k}}\right)
$$


where $N(x)$ is a matrix called shape function defined by:

$$
N(x)=\boldsymbol{p}^{T}(x) \boldsymbol{C}\left(\boldsymbol{X}^{\boldsymbol{k}}\right),
$$

with $C\left(X^{k}\right)=A^{-1}\left(X^{k}\right) B\left(X^{k}\right)$. Note that according to the least square nature of the approximation, $u(x) \cong \hat{u}(x) \neq \lambda(x)$. That is, the local values of the approximating function do not fit the nodal unknown values. Indeed, $\hat{u}(x)$ is the true approximation, which we will use to satisfy the differential equation and the boundary conditions; in this context, $\lambda(x)$ are simply the unknown parameters we aim to determine. According to the concepts described above and Eq. (9), it is possible to obtain the following expressions.

$$
\hat{u}_{x}(x)=\boldsymbol{N}_{x}^{T}(x) \boldsymbol{\lambda}\left(\boldsymbol{X}^{\boldsymbol{k}}\right) \quad \text { and } \quad \hat{u}_{x x}(x)=\boldsymbol{N}_{x x}^{T}(x) \boldsymbol{\lambda}\left(\boldsymbol{X}^{\boldsymbol{k}}\right),
$$

where $(\cdot)_{x}$ and $(\cdot)_{x x}$ denote the first and the second space derivatives, respectively. Note that these derivatives are computed by taking the derivative of the basis functions $\boldsymbol{p}(x)$ in Eq. (2).

\section{The nonlocal damage model}

The so called continuum damage model have been used thoroughly to simulate the behavior of materials that present degradation of the mechanical properties due to small fissures that appears during the loading process. To characterize this, the concept of effective stress $\bar{\sigma}$ is introduced. In one dimension we can write

$$
\sigma=(1-d) \bar{\sigma}
$$

Where $\bar{\sigma}$ is the effective stress and $d$ is the damage parameter between $0 \leq \mathrm{d} \leq 1$. The stress with the effective deformation $\varepsilon$ can be related by Hooke's law:

$$
\bar{\sigma}=E \varepsilon
$$

Where $\mathrm{E}$ is the elastic modulus of the material, then replace (12) in (13) we have:

$$
\sigma=(1-d) E \varepsilon \quad 0 \leq d \leq 1
$$

for the nonlocal damage model based on non-local displacements [26], equation 14 takes the following form

$$
\sigma\left(\varepsilon_{a}, \varepsilon_{g}\right)=\left(1-d\left(\varepsilon_{g}\right)\right) C: \varepsilon_{a}
$$

where $\varepsilon_{g}=\nabla u_{g}$ is the gradient-enriched strain and $\varepsilon_{a}=\nabla u_{a}$ is the strain associated to the local displacement field $u_{a}$. also the displacement field $u_{g}$ is the solution of the diffusion-reaction equation (16) in which $u_{a}$ is the source term

$$
u_{g}-l^{2} \nabla^{2} u_{g}=u_{a}
$$

with the folllowing boundary conditions

$$
\begin{aligned}
u_{g} \cdot n=u_{a} \cdot n & \text { on } \Gamma_{u} \\
\nabla\left(u_{g} \cdot t\right) \cdot n=\nabla\left(u_{a} \cdot t\right) \cdot n & \text { on } \Gamma_{t}
\end{aligned}
$$


the damage parameter $d\left(\varepsilon_{g}\right)$,depends on the non-local strain $\varepsilon_{g}$ via the history variable $\kappa$, defined as

$$
\kappa(t)=\max _{\tau \leq t} Y(t)
$$

where $Y$ is a scalar state variable. In one dimensional setting, $\mathrm{Y}$ is simply the scalar strain $\varepsilon_{g}$. In a milti-dimensional setting, $Y$ is defined either as

$$
Y=\sqrt{\sum_{i} \max \left(0, \varepsilon_{i}\right)}
$$

where $\varepsilon_{i}$ are the principal strains of $\varepsilon_{g}$ or as

$$
Y=\frac{k-1}{2 k(1-2 v)} I_{1}+\frac{1}{2 k} \sqrt{\left(\frac{k-1}{1-2 v} I_{1}\right)^{2}+\frac{12 k}{(1+v)^{2}} J_{2}}
$$

where $I_{1}$ and $J_{2}$ are the first invariant and the second desviatoric invariant respectively, $v$ the Poisson's ratio and $k$ the ratio of compressive to tensile strength.

In this work we use a linear evolution law for the damage parameter

$$
d(\kappa)=\left\{\begin{array}{cl}
0 & \text { if } \kappa \leq k_{i} \\
\frac{k_{u}\left(\kappa-k_{i}\right)}{\kappa\left(k_{u}-k_{i}\right)} & \text { if } k_{i}<\kappa \leq k_{u} \\
1 & \text { if } \kappa>k_{u}
\end{array}\right.
$$

where $k_{i}$ is the damage initiation strain and $k_{u}$ is the ultimate strain. This model is suitable for quasi-brittle materials.

\section{Numerical implementation}

Considerer the system of differential equations which governs the behavior of a solid

$$
\begin{array}{rr}
\nabla \sigma(\mathbf{x})+\rho \mathbf{b}(\mathbf{x})=0 & \forall \mathbf{x} \in \Omega \\
\sigma(\mathbf{x}) \cdot \hat{\mathbf{n}}=\overline{\mathbf{t}}(\mathbf{x}) & \forall \mathbf{x} \in \Gamma_{t} \\
\mathbf{u}(\mathbf{x})=\hat{\mathbf{u}}(\mathbf{x}) & \forall \mathbf{x} \in \Gamma_{u}
\end{array}
$$

using the point collocation method, we can obtain a discrete system of equations [20]

$$
\begin{aligned}
\mathbf{L}^{\mathbf{T}} \sigma+\rho \mathbf{b}=0 & \forall \mathbf{x} \in \Omega \\
\mathbf{n}^{\mathbf{T}} \sigma=\overline{\mathbf{t}} & \forall \mathbf{x} \in \Gamma_{t} \\
\mathbf{u}=\hat{\mathbf{u}} & \forall \mathbf{x} \in \Gamma_{u}
\end{aligned}
$$

where $\mathbf{L}$ is an operator that defines the differential equation, $\mathbf{n}$ contains the normal direction on the external contour, $\sigma$ is the stress vector, $\mathbf{u}$ is the displacement vector, $\rho \mathbf{b}$ the body forces, $\overline{\mathbf{t}}$ and $\hat{\mathbf{u}}$ prescribed force and displacement on the external contour.

To obtain an equivalent system in term of displacement, use the stress-strain relation and the strain-displacement relationships as follows [29].

$$
\sigma=\left(\mathbf{I}-\mathbf{d}\left(\varepsilon_{\mathbf{g}}\right)\right) \mathbf{C} \varepsilon_{\mathbf{a}} \quad \text { en donde } \quad \varepsilon_{\mathbf{a}}=\mathbf{N}_{\mathbf{x}} \mathbf{u}_{\mathbf{a}}
$$




$$
\begin{gathered}
{\left[(\mathbf{I}-\mathbf{d}) \mathbf{L}^{T} \mathbf{C L N}+(\mathbf{I}-\mathbf{d})(\mathbf{L N})^{\mathbf{T}} \mathbf{C L N}-(\mathbf{L N d})^{T} \mathbf{C L N}\right] \mathbf{u}^{h}=-\rho \mathbf{b} \forall \mathbf{x} \in \Omega \text { (30a) }} \\
{\left[\mathbf{n}^{T} \mathbf{C L N}\right] \mathbf{u}^{h}=\overline{\mathbf{t}} \quad \forall \mathbf{x} \in \Gamma_{t}} \\
{[\mathbf{N}] \mathbf{u}^{h} \quad=\overline{\mathbf{u}} \quad \forall \mathbf{x} \in \Gamma_{u}}
\end{gathered}
$$

with its compact form

$$
\mathbf{K}_{d I} \mathbf{u}^{h}=\mathbf{f}_{I} \quad I=1, \ldots ., N
$$

Where $\mathbf{K}_{\mathbf{d}}$ is the stiffness matrix in FPM and $\mathbf{f}$ contain the equilibrium and boundary conditions.

\subsection{Approximation of diffusion-reaction equation}

consider the numerical implementation obtained in (31) and the diffusion-reaction equation (16), to approximate the diffusion term, we used the second-order shape function directly in the same way as in the lineal elastic behavior, then we can write the following stiffness matrix equation for the non-local problem

$$
\mathbf{K}_{\mathbf{N L} I}=\left(\begin{array}{cc}
\mathbf{K}_{\mathbf{d} I} & \mathbf{0} \\
-\mathbf{N} & \left(\mathbf{N}-l_{c}^{2} \mathbf{D}\right)
\end{array}\right)
$$

where $\mathbf{D}$ is the diffusivity matrix defined as

$$
\mathbf{D}=\sum_{k=1}^{i} \frac{\partial^{2}()}{\partial k^{2}}=\sum_{k=1}^{i} N_{k k}()
$$

and the compact form

$$
\mathbf{K}_{\mathrm{NL} I} \mathbf{u}^{\mathbf{h}}=\mathbf{f}_{I}
$$

In this case $\mathbf{u}^{\mathbf{h}}=\left(\mathbf{u}_{\mathbf{a}}^{\mathbf{h}} \mathbf{u}_{\mathbf{g}}^{\mathbf{h}}\right)^{T}$ and $\mathbf{f}$ contain the conditions for the equilibrium and the diffusionreaction equation.

\section{Numerical examples}

For the examples to develop we considered a regular points discretization. The number of points in the interpolation clouds will be specific to each problem. 
Table 1. Uniaxial tensile test

\begin{tabular}{|l|l|l|}
\hline Description & Symbol & Value \\
\hline Length of the bar & $L_{0}$ & 100 \\
Length of weaker part & $l i$ & 10 \\
Cross-section of bar & $A$ & 1 \\
Youn's modulus & $E$ & 20000 \\
Damage threshold & $\epsilon_{0}$ & $10^{-4}$ \\
Final strain & $\epsilon_{f}$ & $1.25 \cdot 10^{-2}$ \\
\hline
\end{tabular}

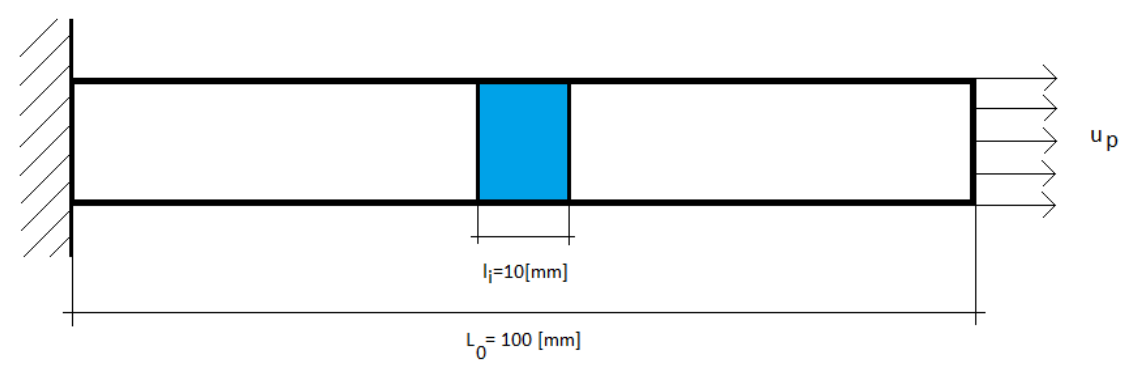

Figure 1. One dimensional rod model

\subsection{Uniaxial tensile test}

The example consists of a rod subjected to uniaxial tensile load by displacement control as show fig. 1. The central tenth of the bar is weakened $10 \%$ by a reduction in Young's modulus to force the localization. The dimensionless geometrical and mechanical parameters are summarized in Table1.

In the first analysis a fixed internal length $l_{c}=\sqrt{5}$ and five different discretization are used, in a second analysis a fixed number of nodes and four different internal length are used. The results in fig. 2 indicates that discretization dependency is removed and fig. 3 show the effects of the internal length size. These results are equivalent to those reported by [26].
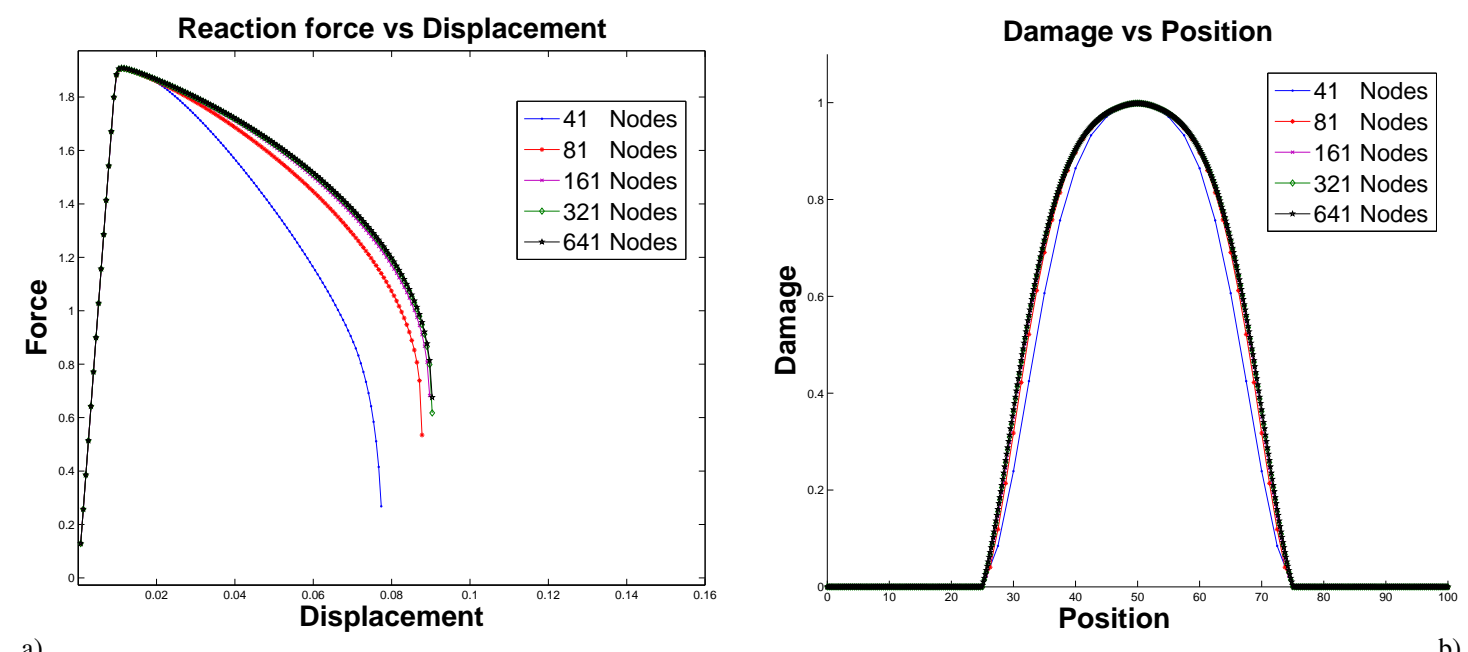

Figure 2. Influence of discretization size on uniaxial tensiale test: a) force-displacement curve .b) damage profile . 

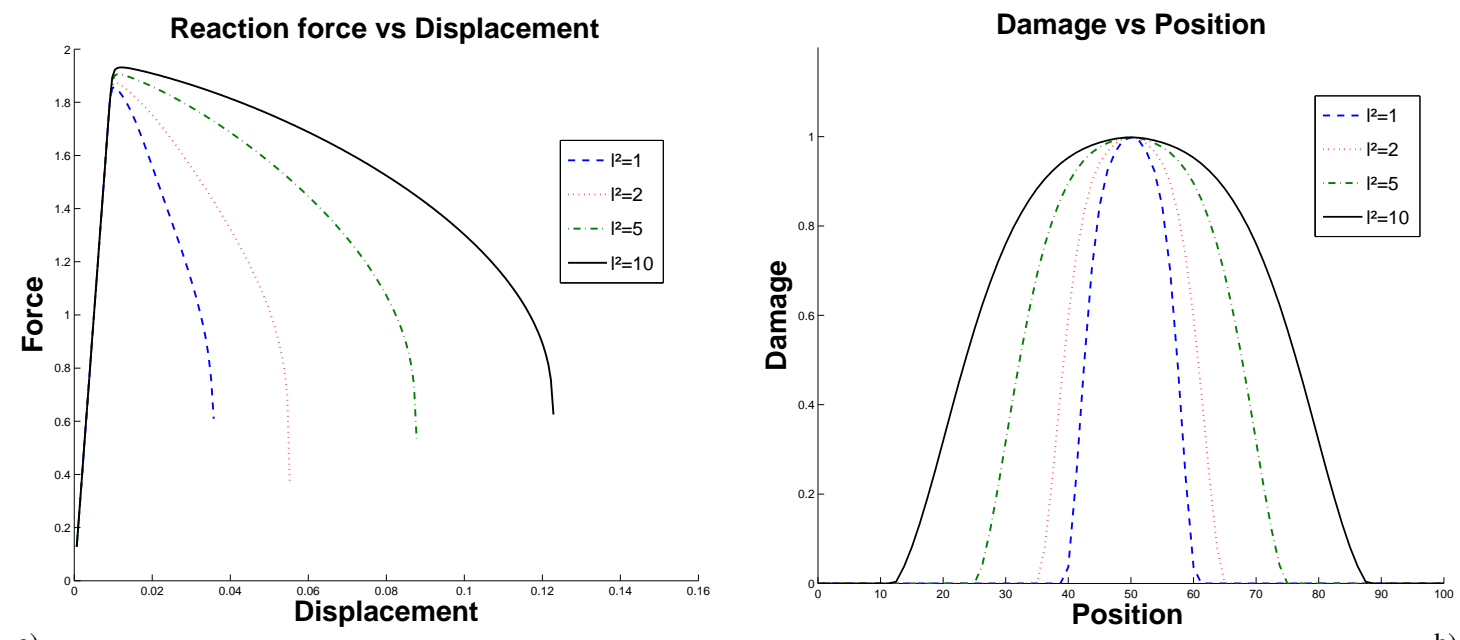

Figure 3. Influence of the internal lenght size on uniaxial tensile test: a) force-displacement curve . b) damage profile .
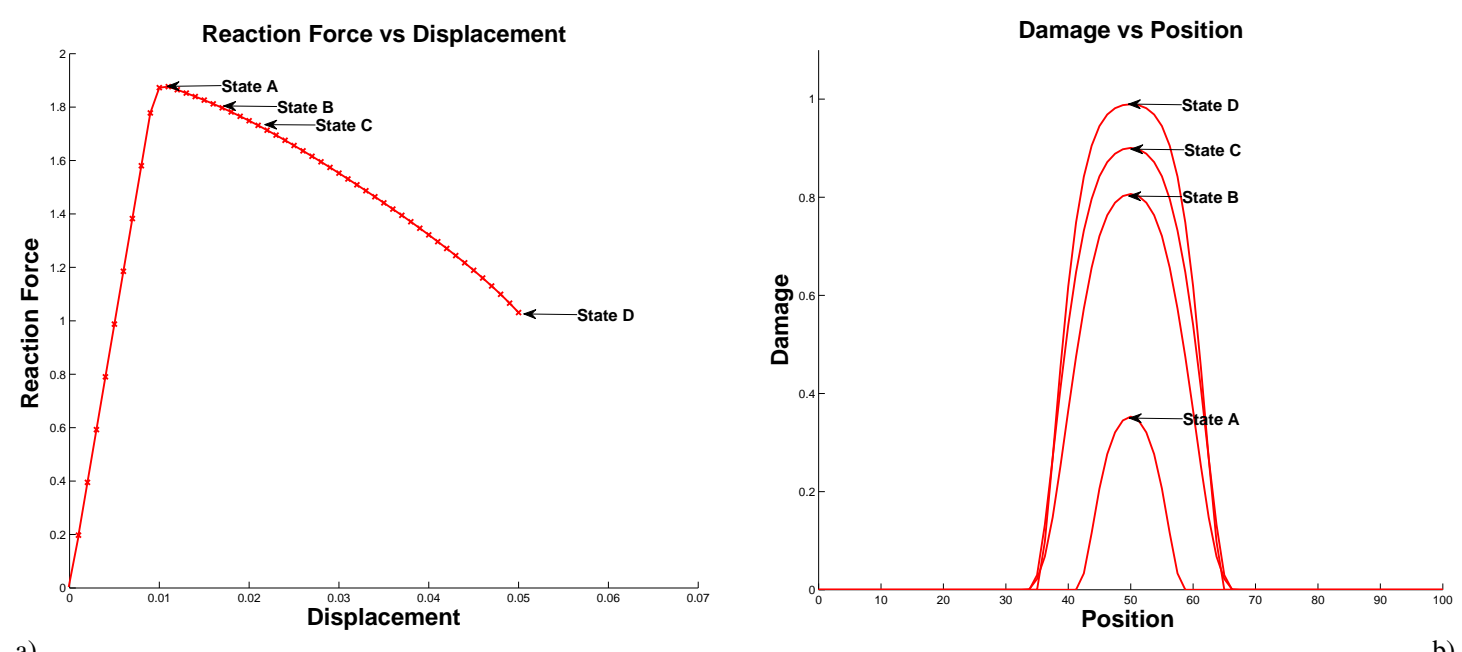

a)

b)

Figure 4. Evolution of the reaction force and internal damage parameter: a) forcedisplacement curve . b) damage profile . 
The final analysis is made with 81 nodes and a internal lenght $l=\sqrt{2}$. The goal is show the evolution of the reaction force, the internal damage parameter and the stress-strain curves for different points.
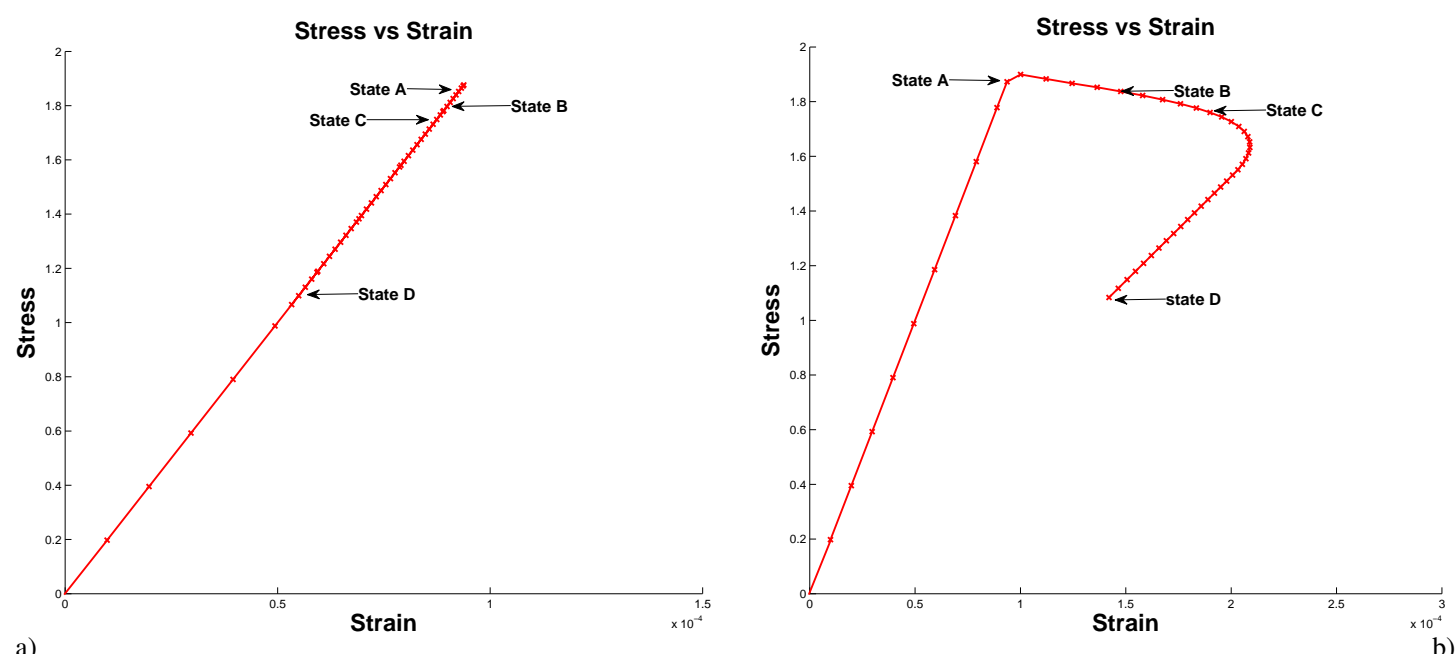

a)

Figure 5. Evolution of the Stress vs Strain at sample points and internal damage parameter: a) $x=0$. b) $x=40$.

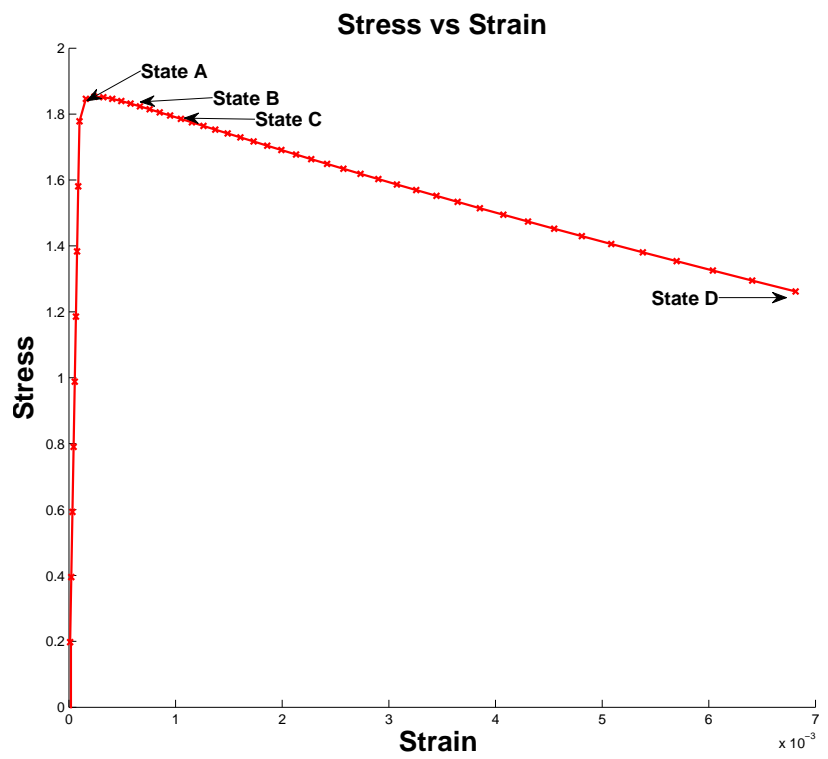

Figure 6. Evolution of the Stress vs Strain at sample points and internal damage parameter at $x=50$

\subsection{Elastic damage tensile specimen with symmetric imperfection}

A rectangular specimen is subjected to uniform displacements at the two ends. In order to trigger localization from a homogeneous state of deformation, symmetric material imperfection with weakened material properties are introduced as show in fig. 7, fig. 8 show the results for different discretizations, these results are equivalent to those reported by [30]. 
The dimensionless material parameters are $E=2 e 6 \nu=0.18 k_{i}=10^{-4} k_{u}=0.00625$ and $l=\sqrt{4.5}$.

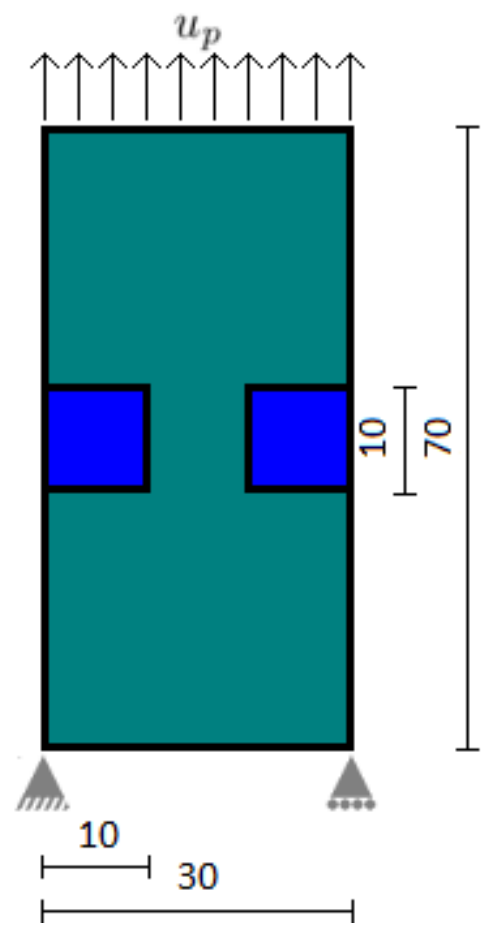

Figure 7. Elastic damage test specimen with symmetric imperfection

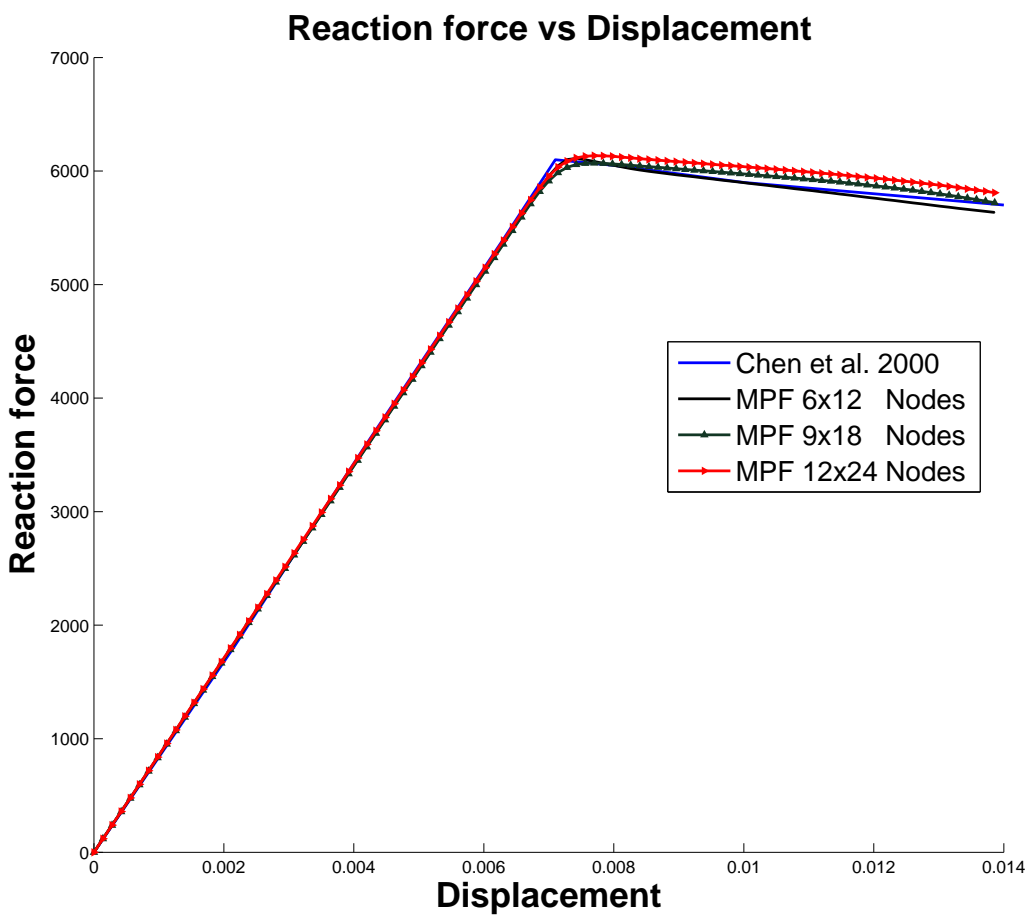

Figure 8. Reaction force vs displacement for various discretizations. 


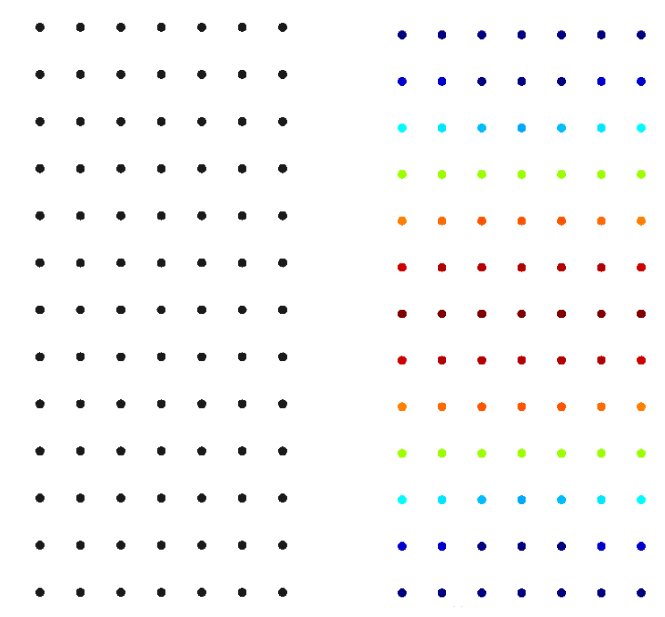

a)

b)

Figure 9. a) analysis model 6x12 nodes . b) strain localization using MPF.

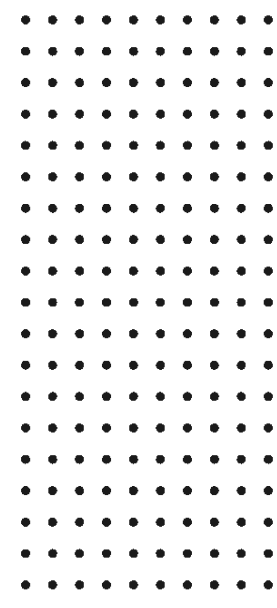

a)

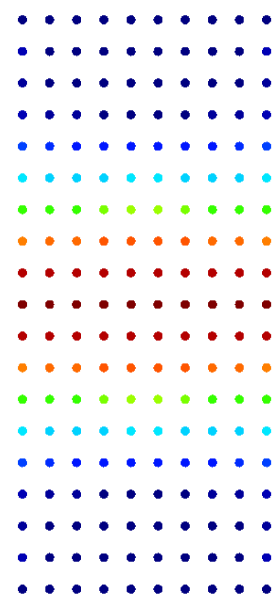

b)

Figure 10. a) analysis model 9x18 nodes. b) strain localization using MPF.

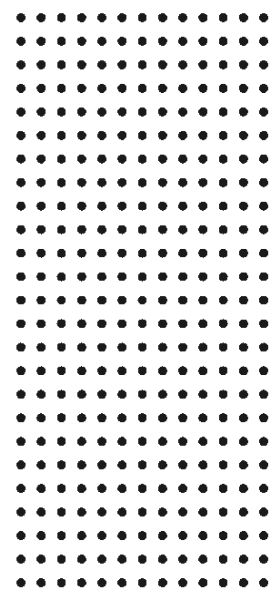

a)

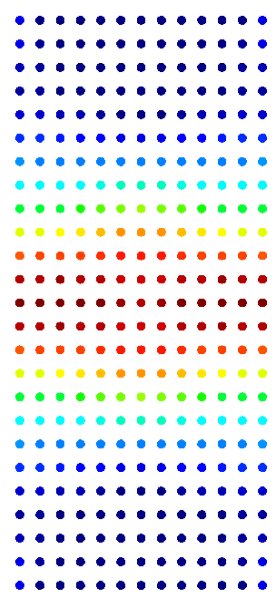

b)

Figure 11. a) analysis model $12 \times 24$ nodes . b) strain localization using MPF. 


\subsection{Elastic damage tensile specimen with asymmetric imperfection}

The principal goal in this example is the study of the shear band formation, an asymmetric imperfection is introduced as show in fig. 12. In this only a refined model is used to examine the proposed method. The material parameters are the same as those used in example 5.2, except $\nu=0.3$ and the internal length $l=\sqrt{0.01}$.

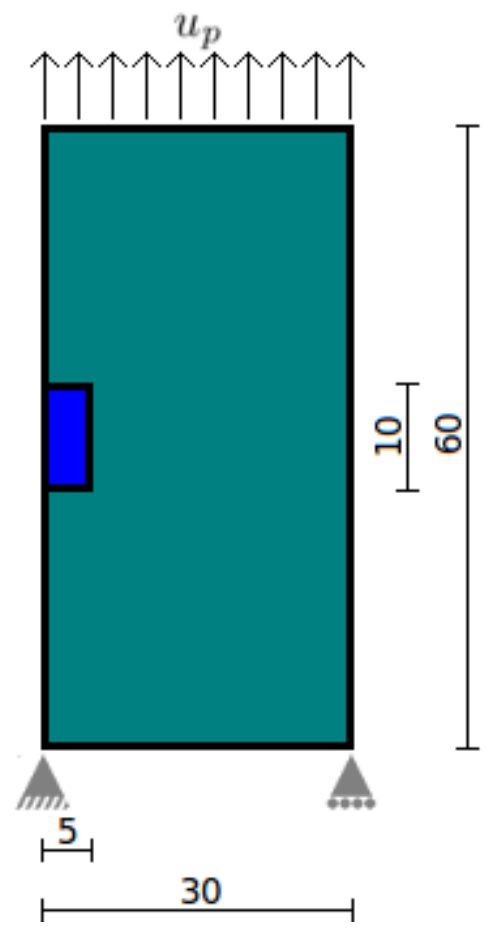

Figure 12. Elastic damage test specimen with asymmetric imperfection

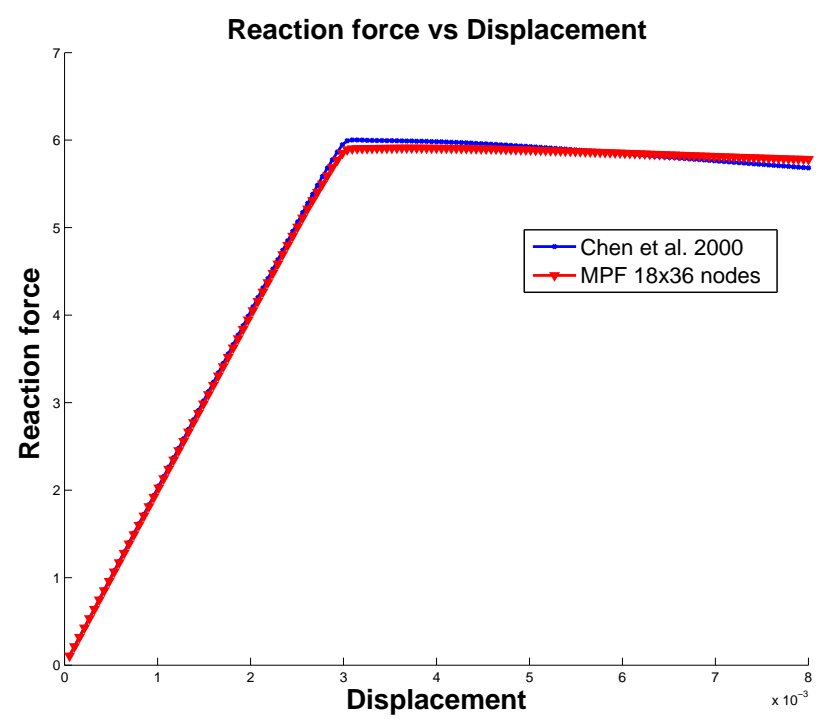

Figure 13. Reaction force vs displacement

Figure 13 show the result with the proposed method, this results is similar to the reported by [30] and fig. 14 shows the shear band formation. 


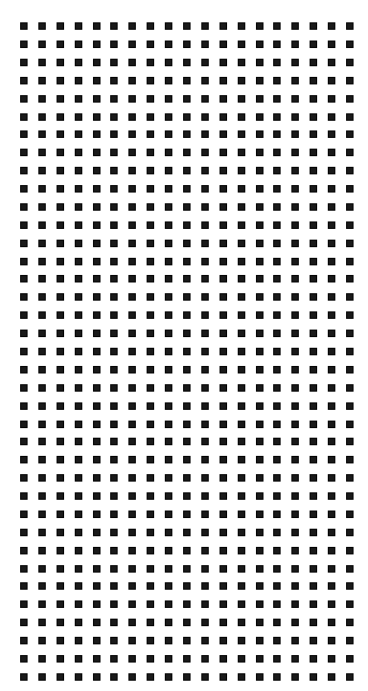

Figure 14. a) analysis model $18 \times 36$ nodes scale x100.

\section{Conclusion}

Has been implemented a isotropic damage model based on nonlocal displacements in strong form by the meshless finite points method to simulate the non-linear material behavior. As the results demonstrate the FPM is able to approximate the localization phenomena with fracture energy regularization by the consideration of characteristic material length and the diffusion-reaction equation. This works concludes the opening of a new alternative viewpoint to the classical weak formulation based on MEF. For a computational aspect, the use of the shape function to approximate all the fields and the simplicity of the development algorithm can be highly attractive.

\section{Acknowledgements}

The authors acknowledge the financial support from the Chilean agency CONICYT (FONDECYT Project 11100253) and UTFSM-DGIP ( PIIC 2011 studentship).

\section{REFERENCES}

\section{References}

[1] T. Fries, H. Matthies, Clasification and overview of meshfree methods, InformatikberichtNr. 2003, Technical University of Braunschweig 3.

[2] S. Li, W. Liu, Meshfree particle methods, Springer, Berlin, 2004. 
[3] Y. Gu, Meshfree methods and their comparisons, International Journal of Computational Methods 4 (2005) 477-515.

[4] Y. Chen, J. Lee, A. Eskandarian, Meshless methods in solids mechanics, Springer, New York, 2006.

[5] E. Oñate, S. Idelsohn, O. Zienkiewics, R. Taylor, C. Sacco, A stabilized finite point method for analysis of fluid mechanics problems, Computer Methods in Applied Mechanics and Engineering 139 (1996) 315-346.

[6] E. Oñate, S. Idelsohn, O. Zienkiewicz, R. Taylor, A finite point methods in computational mechanics, aplication to convective transport and fluid flow, International Journal for Numerical Methods in Engineering 39 (1996) 3839-3866.

[7] E. Oñate, S. Idelsohn, A mesh free finite point method for advective-diffusive transport and fluid flow problems, Computational Mechanics 21 (1998) 283-292.

[8] E. Oñate, C. Sacco, S. Idelsohn, A finite point method for incompressible flow problems, Computer Visual Science 3 (2000) 67-75.

[9] E. Oñate, F. Perazzo, J.Miquel, A finite point method for elasticity problems, Computer and Structures 79 (2001) 2151-2163.

[10] F. Perazzo, S. Oller, J. Miquel, E. Oñate, Avances en el método de puntos finitos para la mecánica de sólidos, Revista Internacional de Métodos Numéricos en Ingeniería 22 (2006) $153-168$.

[11] F. Perazzo, J. Miquel, E. Oñate, El método de puntos finitos para problemas de la dinámica de sólidos, Revista Internacional de Métodos Numéricos en Ingeniería 20 (2004) 235-246.

[12] L. Zhang, Y. Rong, H. Shen, T. Huang, Solidification modeling in continuous casting by finite point method, Journal of Materials Processing Technology 192-193 (2007) 511-517.

[13] L. Pérez-Pozo, F. Perazzo, Non-linear material behaviour analysis using meshless finite point method, in: 2nd ECCOMAS Thematic Conference on Meshless Methods, Porto, Portugal, 2007, pp. 251-268.

[14] L. Pérez-Pozo, F. Perazzo, A. Angulo, A meshless fpm model for solving nonlinear material problems with proportional loading based on deformation theory, Advances in Engineering Software 40 (2009) 1148-1154.

[15] F. Perazzo, R. Lohner, L. Perez-Pozo, Adaptive methodology for meshless finite point method, Advances in Engineering Software 22 (2007) 153-168.

[16] A. Angulo, L. Pérez-Pozo, F. Perazzo, A posteriori error estimator and an adaptive technique in meshless finite point method, Engineering Analysis with Boundary Elements 33 (2009) 1322-1338. 
[17] M. Bitaraf, S. Mohammadi, Large deflection analysis of flexible plates by the meshless finite point method, Thin-Walled Structures 48 (2010) 200-214.

[18] J. Peraire, J. Peiro, L. Formaggia, K. Morgan, O. Zienkiewicz, Finite element euler computations in three dimensions, International Journal for Numerical Methods in Engineering 26 (1988) 2135-2159.

[19] E. Ortega, E. Oñate, S. Idelsohn, An improved finite point method for three-dimensional potential flows, Computational Mechanics 40 (2007) 949-963.

[20] F. Perazzo, Una metodología numérica sin malla para la resolución de las ecuaciones de elasticidad mediante el método de puntos finitos, Universitat Politécnica de Cataluña, Barcelona España, 2002, tesis Doctoral.

[21] Martin A. Análisis y formulación de un estimador del error en el método sin malla de punto finites. Universidad Técnica Federico Santa María, Valparaíso Chile, 2006 Trabajo de titulo.

[22] R. Taylor, S. Idelsohn, O. Zienkiewicz, E. Oñate, Moving least square approximations for solution of differential equations, CIMNE Research Report 74.

[23] Lemaitre J. and J. L. Chaboche. Mechanics of solid materials. Cambridge University Press.

[24] Hilleborg A., M. Modeer, and P.A. Petterson. Analysis of crack formation and crack growth in concrete by means of fracture mechanics and finite elements. Cement and concrete research.6, 773-782.

[25] Oliver J. A. E. Huespe, M. D. Pulido, and E. Chaves . From continuum mechanics to fracture mechanics: the strong discontinuity approach. Engineering Fracture Mechanics 69(2), 113-136.

[26] Rodríguez-Ferran A., I. Morata , and A. Huerta. A new damage model based on nonlocal displacements. International Journal for numerical and analytical methods in geomechanics 29(5), 473-493.

[27] Bazant, Z. P. and B. H. Oh. Crack band theory for fracture in concrete. Materials and structures 16(3),155-177.

[28] Pérez-Pozo L, Campos A. Regularización de la localización por medio de gradients de deformación plastic no local z el método sin malla de puntos finites.

[29] Pérez-Pozo L, Chacana F. Avances en la reguarización de la energía de gracira en un modelo de daño isotropic mediante un método sin malla. CILAMCE 2011.

[30] Chen J., Wu C., Belytschko T. Regularization of material instabilities by meshfree approximations with intrinsic lenght scales. Int. Journal for numerical methods in engineering. 